\title{
PROCEEDING
}

\section{Proceedings of the 30th annual symposium of the Society for the Study of Delivery Management: Prolegomena}

\author{
Katsuhiko Naruse \\ Department of Obstetrics and Gynecology, Nara Medical University
}

The Society for the Study of Delivery Management (SSDM) was established over 30 years ago and recently held its annual symposium during the annual meeting of the Japan Society for the Study of Hypertension in Pregnancy (JSSHP). To engage both the SSDM and JSSHP, Secretary Generals of the JSSHP (K. Naruse and Dr. Shintaro Makino from Juntendo Univ., Tokyo) were requested by Dr. Katsuto Eguchi (President of the JSSHP, Okayama Central Hospital, Okayama) and Dr. Shigeru Saito (President of the Meeting and President-elect of the JSSHP, Toyama Univ., Toyama) to state the new aims of the SSDM symposium.

Recently, new research from studies involving topics such as uterine spiral artery remodeling and epigenetic changes has proposed the direct effects as well as the pathophysiological similarities of pregnancy induced hypertension (PIH) as compared to other emergent obstetric diseases such as placental abruption, fetal growth restriction and non-reassuring fetal status, thrombotic diseases, amniotic fluid embolism, or brain stroke. However, very few groups in Japan and elsewhere accept the viewpoints of researchers/clinicians who wish to discuss obstetric emergency diseases from a broader perspective which includes early placentation failure, genetic changes that link PIH and other issues, and failed cases of PIH leading to other organ failure. As such, we propose addressing "obstetric emergencies" as a new aim for our annual symposium over the next few years. This will comprise one portion of the JSSHP, but will not be strongly restricted to a discussion on hypertension.

This year, we invited five clinicians to the symposium on October 5, 2013 in Toyama, chaired by Dr. Jun Yoshimatsu (National Cerebral and Cardiovascular Center, Osaka) and Dr. Koichiro Shimoya (Kawasaki Univ., Okayama). The theme was "Infusion-andoutput balance on PIH and other obstetric emergency (include blood transfusion and control of postpartum hemorrhage)," and a fruitful discussion developed among the attendees, who comprised many passionate and enthusiastic young doctors and residents. The aim of the future SSDM remained undecided and is currently under revision by the next President of the Meeting (Dr. Satoru Takeda, Juntendo Univ., Tokyo), but the general consensus was to invite active researchers and clinicians to give talks with empirical data as well as findings on useful, challenging, or innovative clinical techniques and outcomes. Further support for this symposium from any clinical or basic research fields is greatly appreciated. 


\section{Peripartum changes in maternal body in twin pregnancies with or without pregnancy induced hypertension and pregnancy-induced antithrombin deficiency}

\author{
Mamoru Morikawa, Takahiro Yamada, Ryutaro \\ Nishida, Rina Akaishi, Takahiro Koyama, \\ Naoto Araki, Takahiro Kojima, Daisuke Endo, \\ Takeshi Umazume, Hisanori Minakami \\ Department of Obstetrics, Hokkaido University Graduate \\ School of Medicine
}

\begin{abstract}
Aim: It is unknown whether maternal weight gain during the last antenatal week and maternal weight loss during the first postpartum month are affected by complications such as pregnancy induced hypertension (PIH) and pregnancy-induced antithrombin deficiency (PIATD).

Methods: Study subjects were 106 women with twin pregnancies who underwent cesarean section. Maternal weight gain during the last antenatal week and net weight loss (maternal weight at delivery minus the sum of neonatal weight, placental weight, blood loss at delivery, and maternal weight at one month postpartum) were examined at one month postpartum. PIATD was defined as perinatal antithrombin activity $\leq 65 \%$ of normal activity following antenatal gradual decline.

Results: Maternal weight gain during the last antenatal week was significantly greater in women with $\mathrm{PIH}$ $(n=10)$ than those without $(n=96) \quad(P=0.046)$. Net maternal weight loss at one month postpartum was also significantly greater in women with $\mathrm{PIH}$ than in those without $(P=0.022)$. Maternal weight change was similarly affected by the presence of PIATD; antenatal weight gain and postnatal weight loss tended to be greater in women with PIATD $(n=19)$ than in those without $(n=87)$, although this difference was not significant.

Conclusions: These results suggest that among women with twin pregnancies, excessive water retention was more likely to occur in the late stage of pregnancy in women with PIH and/or PIATD.
\end{abstract}

Management of Postpartum Hemorrhage: Fibrinogen Supplementation and Transcatheter Arterial Embolization

\author{
Koichi Shinohara, Chiharu Kimura, Toshitaka \\ Mori, Kazushi Watanabe, Akihiko Wakatsuki \\ Department of Obstetrics and Gynecology, Aichi Medical \\ University, School of Medicine
}

Fibrinogen plays a very important role in achieving and maintaining hemostasis and is necessary for effective clot formation. As such, fibrinogen is a key target for the treatment and prevention of postpartum hemorrhage. Fibrinogen is the first coagulation factor, and if its levels drop to critical levels $(<100 \mathrm{mg} / \mathrm{dl})$ during a major hemorrhage, the bleeding may become uncontrollable, even with transcatheter arterial embolization. Current guidelines recommend maintaining plasma fibrinogen levels above $150 \mathrm{mg} / \mathrm{dl}$.

Fibrinogen can be acquired from fresh frozen plasma. However, if kept in frozen form, roughly 30 minutes of preparation time is required prior to use. Fibrinogen concentrate, on the other hand, is advantageous in that it allows for a standardized dose of fibrinogen to be rapidly administered in a small volume, and it has a very good safety profile. In this presentation, we highlight the importance of maintaining plasma fibrinogen levels and supplementation of fibrinogen. We have found that fibrinogen concentrate is associated with a reduction in or even total elimination of allogeneic blood product transfusion. Fibrinogen concentrate represents an important option for the treatment of coagulopathic bleeding. Thus, at our hospital, we have enabled the immediate selection of rapid administration of cryoprecipitate and fibrinogen concentrate. This study has been approved by the Ethics Committee of Aichi Medical University. Further studies are needed to determine precise dosing strategies and thresholds for fibrinogen supplementation.

Key words: blood transfusion, fibrinogen concentrate, hemostasis, obstetrics, postpartum hemorrhage 


\section{Initial report of a multicentre case series of recombinant activated factor VII (rFVIIa / NovoSeven) in Japan}

\author{
Takao Kobayashi \\ Hamamatsu Medical Center
}

Background: The off-label use of recombinant activated factor VII (rFVIIa) in patients with life-threatening bleeding has been reported worldwide to be effective in various clinical settings; however, only limited numbers of case reports documenting the use of rFVIIa in Japanese patients with postpartum hemorrhage have been published to date. Data on Japanese cases with severe postpartum hemorrhage in which rFVIIa had been administered were collected.

Methods: Data from patients with obstetric hemorrhage who were treated with rFVIIa between 2005 and 2010 were retrospectively collected throughout Japan. Participating institutions obtained approval from their ethics committees to collect relevant patient data retrospectively without disclosure of personally identifiable information to third-party organizations.

Results: A total of 25 women received rFVIIa for the treatment of obstetric hemorrhage at 18 institutions. The primary causes of massive bleeding for which rFVIIa was given included uterine atony, placental abruption, placenta previa/increta, uterine rupture, and preeclampsia, among others. On average, total estimated blood loss was 14,032 $\mathrm{ml}$, and median blood loss was $9,781 \mathrm{ml}$ (range: 2,198-55,660 $\mathrm{ml}$ ). After the final administration, bleeding was stopped in 16 patients $(64 \%)$, decreased in 8 patients $(32 \%)$, and remained unchanged in 1 patient $(4 \%)$. A significant reduction in blood product requirement was observed following the first rFVIIa administration. Hysterectomy was required in 13 patients (52\%). Regarding hematologic parameters before rFVIIa administration, the median fibrinogen level was $145 \mathrm{mg} /$ $\mathrm{dl}$ and the median platelet level was $8.5 \times 10^{9} / 1$ in cases where bleeding was stopped. Four asymptomatic thrombotic events were reported in 3 patients.

Conclusions: These results suggest that rFVIIa can be a beneficial therapeutic option that can reduce blood loss and prevent hysterectomy in Japanese patients with massive obstetric bleeding.

Key words: hysterectomy, postpartum hemorrhage, recombinant activated factor VII ( $r F V I I a)$

\section{The history before the approval of the Bakri uterine balloon and its future prospects}

\author{
Shintaro Makino, Takashi Yorifuji, Chihiro Hirai, \\ Tetsunori Inagaki, Tomomi Saito, \\ Yuka Yamamoto, Atsuo Itakura, Satoru Takeda
}

Department of Obstetrics and Gynecology, Juntendo Faculty of Medicine

Hemostasis of massive postpartum hemorrhage is addressed by 4 major methods: (1) administration of a uterotonic agent, (2) coagulation factor replacement, (3) emergency temporary hemostasis (including bimanual compression, intrauterine balloon tamponade, and gauze packing), and (4) additional hemostatic procedures (compression suture, transarterial embolization, and hysterectomy). Methods 1 and 3 are performed almost simultaneously. Methods 2 and 4 are performed when hemostasis is not achieved or blood loss does not decrease, and these methods might be necessary in patients with elevated indicators of shock or those with DIC.

In 1985, Bowen et al. reported on its effectiveness against postpartum hemorrhage. In 1992, Bakri reported on the effectiveness of uterine tamponade for placenta previa accreta. In recent years, Georgiou published a report on 106 patients with postpartum hemorrhage in whom hemostasis was achieved at the high rate of $91.5 \%$. The use of Bakri balloons is recommended in clinical practice guidelines recommended by the WHO, FIGO, ACOG, RCOG, and SOGC. However, Bakri balloons have only been on the Japanese market since April 2013, which is eleven years after they were on the U.S. market (April 2002). The Bakri balloon is now approved as a special medical material covered by health insurance. Thus, its wide use can be expected within a short time as a standard care measure. 